SHORT REPORT

\title{
Linear scleroderma "en coup de sabre" coexisting with plaque-morphea: neuroradiological manifestation and response to corticosteroids
}

\author{
I Unterberger, E Trinka, K Engelhardt, A Muigg, P Eller, M Wagner, N Sepp, G Bauer
}

J Neurol Neurosurg Psychiatry 2003;74:661-664

A 24 year old woman in the 33rd week of pregnancy developed progressive neurological complications with right sided hemiparesis in association with the occurrence of linear scleroderma "en coup de sabre" (LSCS) and preexisting plaque-morphea, already being treated by balneophototherapy. Further progression of neurological symptoms led to a caesarean section with the delivery of a healthy child. Brain magnetic resonance imaging (MRI) showed focal T2 signal increases in the left frontoparietal region directly adjacent to the area of LSCS. Cerebrospinal fluid analysis revealed oligoclonal bands, suggesting an intracerebral inflammatory process. Subsequent pulsed corticosteroid treatment led to a remission of neurological symptoms and to a marked resolution of the MRI lesions.

L near scleroderma "en coup de sabre" (LSCS) and plaquemorphea are both variants of localised scleroderma. ${ }^{1}$ LSCS - presents as band-like sclerotic skin lesions, usually involving a single unilateral change in the frontoparietal area of the head. This is characterised by atrophy and a furrow of the skin. Progressive involution of the craniofacial bones may result in hemifacial atrophy, which appears to be identical to the Parry Romberg syndrome (idiopathic progressive facial hemiatrophy). A clear differentiation between Parry Romberg syndrome and LSCS is often not possible and the aetiology of the two conditions may be similar. ${ }^{2}$

Plaque-morphea is characterised by circumscribed sclerotic plaques with ivory coloured centres and, in the active state of the disease, with violaceous borders. With further progression the centre becomes white or yellowed. Most commonly the lesions are single or few in number, but they may be multiple and are typically localised on the trunk and the extremities.

Compared with plaque-morphea, LSCS may be accompanied by neurological complications such as epileptic seizures and other focal neurological symptoms. ${ }^{2-7}$ Ipsilateral abnormalities of brain imaging have been reported in patients with LSCS. $^{2}{ }^{45-9}$

We report an unusual case of a pregnant woman with preexisting plaque-morphea, newly developed LSCS associated with progressive neurological symptoms, and a rapid response to pulsed corticosteroid treatment.

\section{CASE REPORT}

A 24 year old woman presented with a two week history of slowly progressive weakness and fine motor disturbances in her right arm. At the age of 20 a plaque-morphea was diagnosed clinically (fig l, panels B and D) and the patient was treated with several courses of balneophototherapy, leading to partial remission of the lesions. There was no progression of skin lesions or any neurological abnormalities during follow up.

On admission the patient was in the 33rd week of pregnancy. She was fully oriented, had normal speech, and presented with a right sided hemiparesis (grade 3/5, MRC scale) with a positive Babinski sign on the right. There was no history of head trauma.

Routine laboratory testing and comprehensive serological screening including antineutrophilic cytoplasmic antibodies (ANCA), anticardiolipin antibodies, and antibodies against Borrelia burgdorferi, was unremarkable except for a mildly increased antinuclear antibody titre (1:160, with a homogeneous pattern). CSF analysis showed a normal cell count ( 7 leucocytes $/ \mathrm{mm}^{3}$ ) and normal protein and glucose, but electrophoresis revealed intrathecal production of IgG and IgM with oligoclonal bands.

Magnetic resonance imaging (MRI) showed multiple lesions with high signal intensity on $\mathrm{T} 2$ weighted images in the left hemisphere, accentuated over the frontoparietal region and involving the white matter with some small hyperintensive subcortical foci over the right frontal regions (fig 2). No significant brain atrophy was found. MRI was done without gadolinium enhanced Tl weighted images because of the pregnancy.

Rapid progression of neurological symptoms prompted a caesarean section with delivery of a healthy child. Further dermatological examination revealed a linear sclerotic atrophic area with hypo- and hyperpigmentation of the left forehead (fig 1, panels A and C), compatible with LSCS, which was confirmed by the typical histopathology on skin biopsy, with fibrosis and sclerosis in the deep regions of the dermis. There was no sign of systemic sclerosis.

Cerebral computed tomography (CCT) done after delivery showed parenchymal calcifications (fig 3 ) in the left frontoparietal regions and skull atrophy directly under the cutaneous lesion. The patient was treated with a pulsed intravenous corticosteroid (methylprednisolone) regimen, $1 \mathrm{~g}$ daily for three days followed by $500 \mathrm{mg}$ daily for further three days, and a consecutive tapering period with oral methylprednisolone. During the following two weeks this treatment led to a rapid improvement in the neurological symptoms with the paresis resolving completely, though the positive Babinski sign remained. Repeat MRI 14 days later showed gadolinium enhanced white matter lesions over the left frontoparietal regions on $\mathrm{Tl}$ weighted images, but the lesions showed marked resolution (fig 4). Because of the rapid clinical improvement we did not undertake a brain biopsy. The patient recovered well and was discharged five weeks after the onset of her neurological symptoms.

\section{DISCUSSION}

Central nervous system involvement in patients with LSCS has been described in several case reports. Patients may 

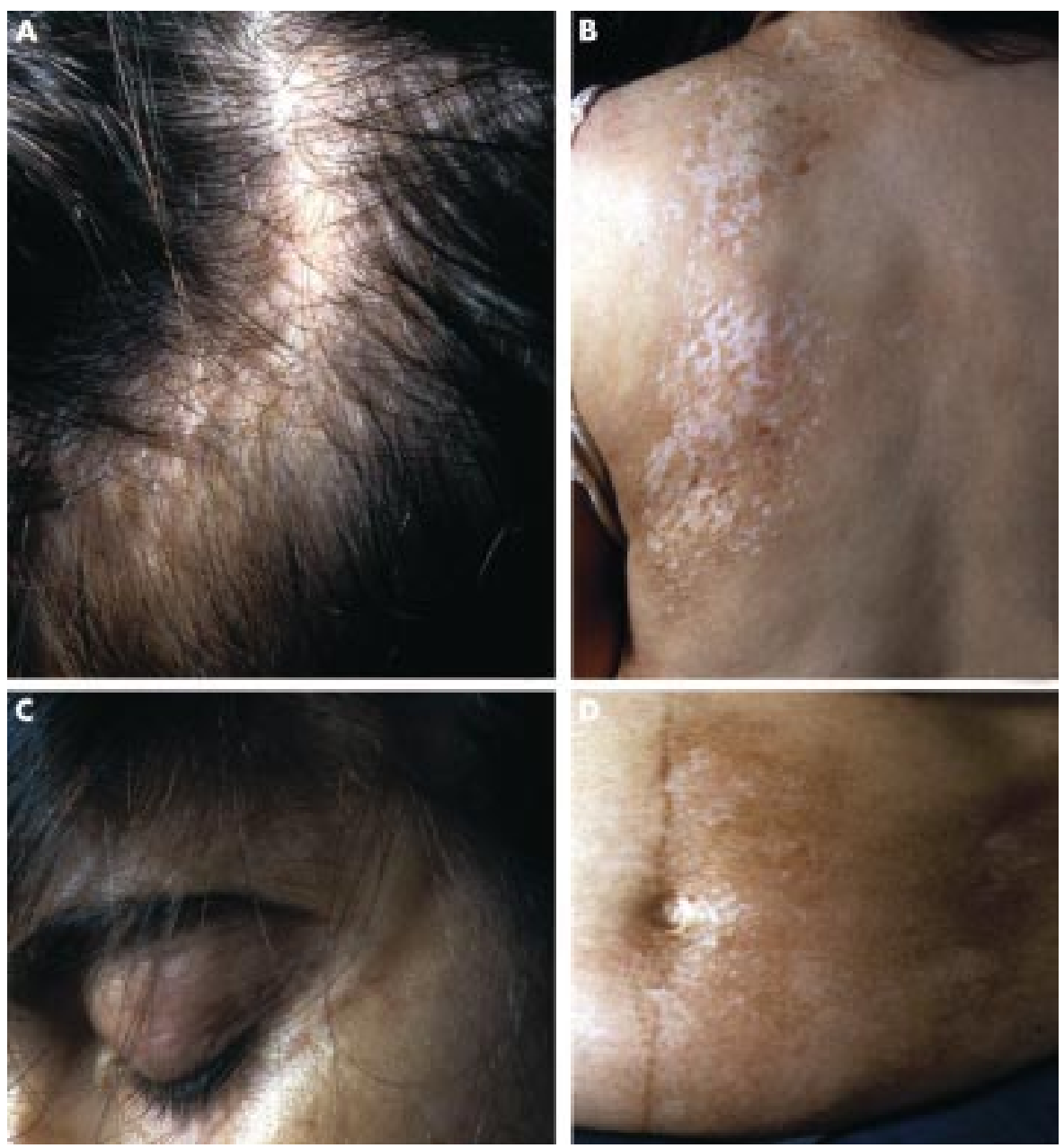

Figure 1 Unilateral furrow of atrophic skin on the frontal part of the capillitium (A) starting in the left periorbial area (C). Irregularly distributed patches of hyper- and hypopigmentated skin, slightly indurated, on the left side of the back (B) and the abdomen (D).

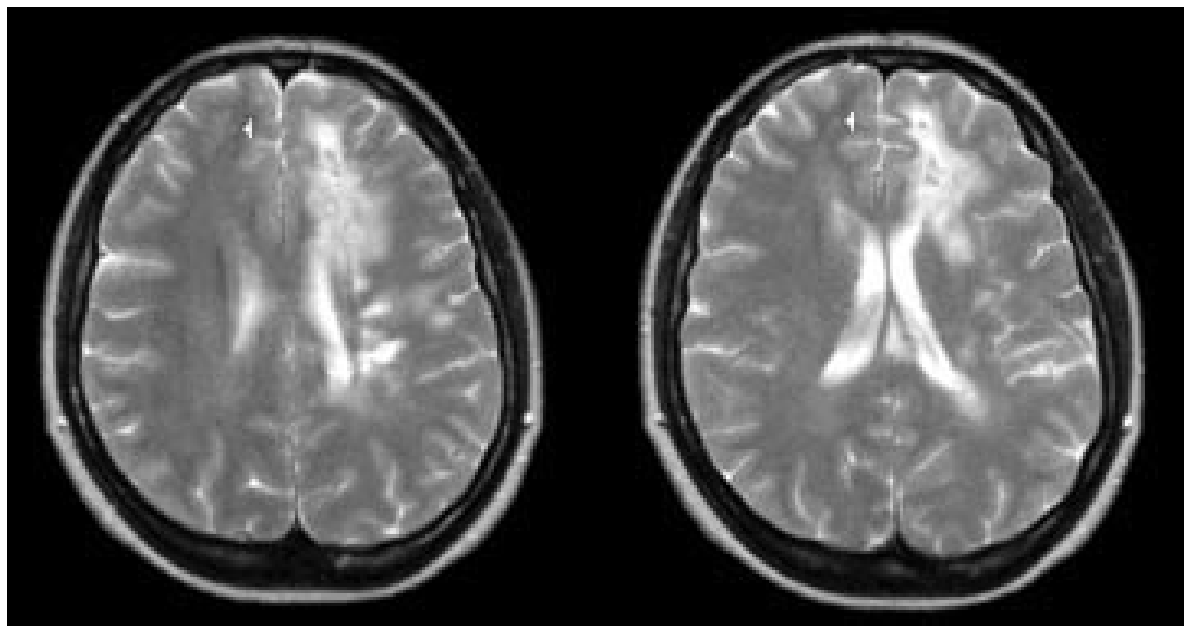

Figure 2 Initial magnetic resonance imaging examination. Axial T2 weighted images showing multiple lesions of high signal predominantly over the left frontoparietal regions involving the white matter. There are some small hyperintensive subcortical foci over right frontal lobe (arrowheads).

present with epileptic seizures ${ }^{2-4} 78$ and other focal neurological symptoms. ${ }^{24511}$ Neuroradiological abnormalities, including cerebral atrophy, white matter lesions, parenchymal calcification, meningeocortical alterations, and skull atrophy, have been described in patients with LSCS. ${ }^{2} 5$ 8-12
Our patient developed progressive right sided hemiparesis during pregnancy. The initial MRI (fig 2) showed multiple white matter lesions over the left frontoparietal regions adjacent to the LSCS, and some small hyperintense subcortical foci over the right frontal regions. Repeat MRI (fig 4), done after a 


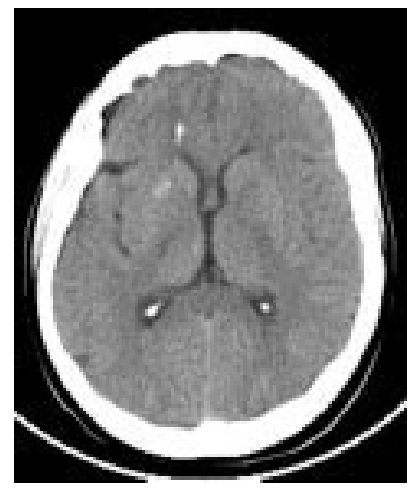

Figure 3 Cerebral computed tomography after delivery, showing parenchymal calcification over the left frontoparietal region and mild skull atrophy directly under the cutaneous lesion.

pulsed corticosteroid treatment, showed marked resolution of the lesions. Computed tomography (fig 3 ) revealed parenchymal calcification in the corresponding area and mild skull atrophy under the skin lesion.

Neuropathological findings in patients with $\operatorname{LSCS}^{27}$ provide evidence for an underlying inflammatory process rather than a vascular dysgenetic origin ${ }^{4}$ as a causative pathogenic pathway. The occurrence of oligoclonal bands in the cerebrospinal fluid, the lesions on MRI, and the response to immunosuppression provide further evidence for this hypothesis. ${ }^{25}$. The MRI findings, the intrathecal production of immunoglobulins, and the excellent response to corticosteroid treatment in our patient support this hypothesis.

Histologically, the skin lesions show perivascular lymphocytic infiltration, proliferation of fibroblasts, and dense collagen deposition within the dermis, and-in contrast to plaque-morphea-the deeper connective tissues including muscle and bone structures may be involved in LSCS. The involvement of all structures including the brain underlying LSCS lesions in our patient is intriguing and suggests an ongoing inflammatory process.

In many patients, linear scleroderma is preceded by local injury ${ }^{713} 14$ which may be a trigger for the underlying inflammatory process. ${ }^{15}$ In our patient there was no history of pre-existing trauma.

To our knowledge there is only one case report describing LSCS in association with plaque-morphea and neurological complications. Menni and coworkers ${ }^{10}$ described two children with both sclerotic and atrophic skin lesions on half of the face and coexisting plaque-morphea, who developed neurological and radiological manifestations years after the onset of the dermatological symptoms. The neurological symptoms resolved spontaneously.
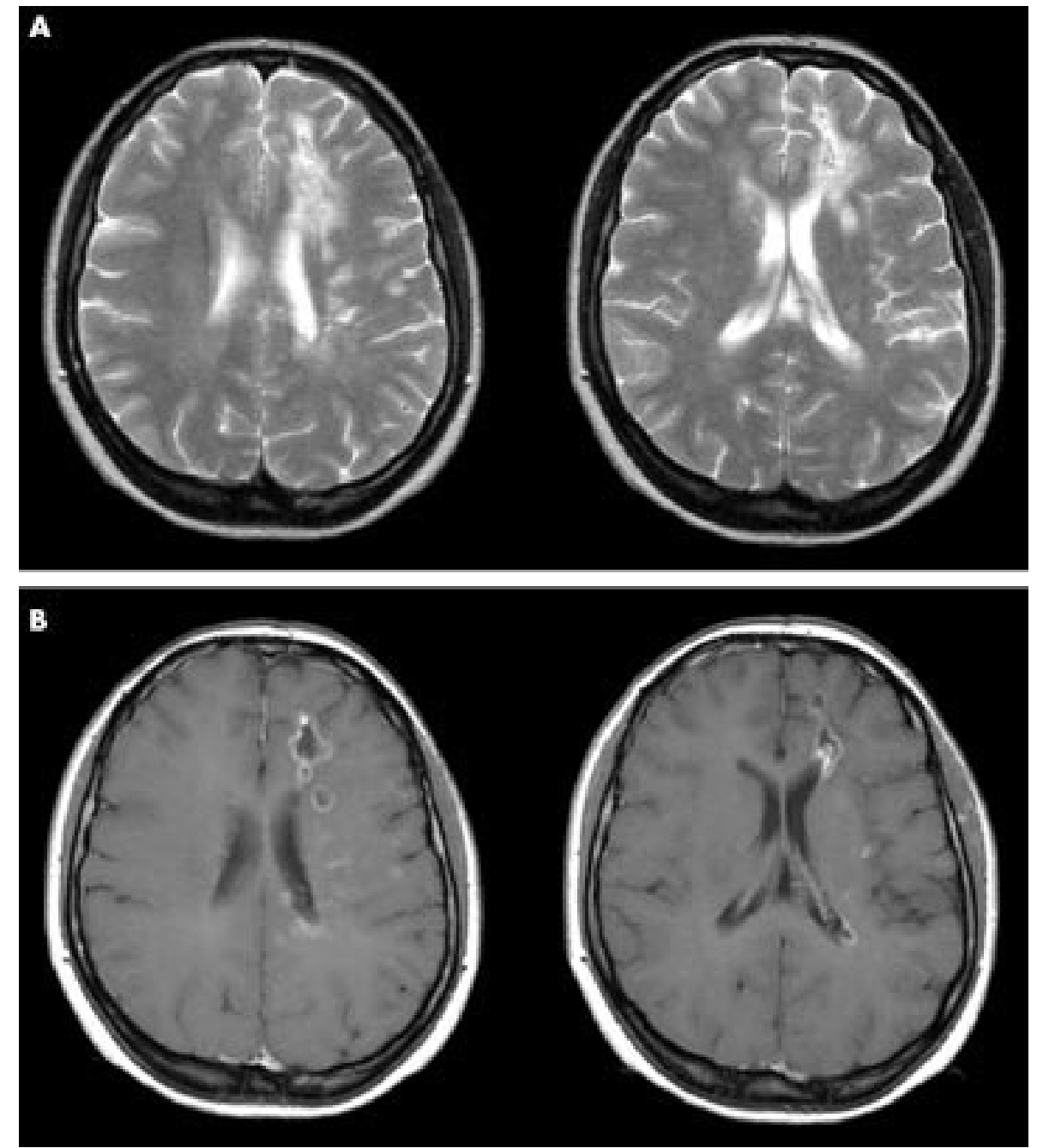

Figure 4 Repeat magnetic resonance imaging after pulsed corticosteroid treatment. (A) Axial T2 weighted images showing marked resolution of the white matter lesions. (B) Axial gadolinium enhanced Tl weighted images showing enhancement of white matter lesions over the left frontoparietal regions. 
The exacerbation of the disease during pregnancy in our patient deserves further comment. The influence of pregnancy on the course of autoimmune diseases is well documented, in particular in patients with systemic lupus erythematosus. ${ }^{16}$ The pathogenesis of both plaque-morphea and LSCS is still unknown; however, autoimmune mechanisms are suspected, leading to the fibrotic changes in skin tissues. ${ }^{17}{ }^{18}$ We therefore speculate that in our patient pregnancy related hormonal changes could have induced further progression of the disease, with the development of LSCS and cerebral involvement.

\section{Authors' affiliations}

I Unterberger, E Trinka, K Engelhardt, A Muigg, P Eller, G Bauer,

Universitätsklinik für Neurologie, Innsbruck, Austria

N Sepp, Universitätsklinik für Dermatologie, Innsbruck

M Wagner, Institut für Magnetresonanztomographie, Innsbruck

Competing interests: none declared

Correspondence to: Dr Unterberger Iris, Universitätsklinik für Neurologie, Anichstrasse 35, A-6020 Innsbruck, Austria; iris.unterberger@uibk.ac.at

Received 6 August 2002

In revised form 14 November 2002

Accepted 16 November 2002

\section{REFERENCES}

1 Rosenwasser TA Eisen AZ Cutaneous manifestations of multisystem diseases. In: Fitzpatrick TB, Eisen AZ, Wolff K, et al, eds. Dermatology in general medicine. New York: McGraw-Hill, 1993:2156-67.

2 Stone J, Franks AJ, Guthrie JA, et al. Scleroderma "en coup de sabre": pathological evidence of intracerebral inflammation. J Neurol Neurosurg Psychiatry 2001:70:382-5

3 Gambichler T, Kreuter A, Hoffmann K, et al. Bilateral linear scleroderma "en coup de sabre" associated with facial atrophy and neurological complications. Biomed Central Dermatol 2001;1:9.
4 Chung $\mathbf{M H}$, Sum J, Morrell $M$, et al. Intracerebral involvement in scleroderma en coup de sabre: report of a case with neurological findings. Ann Neurol 1995;37:679-81.

5 Luer W, Jöckel D, Henze T, et al. Progressive inflammatory lesions of the brain parenchyma in localized scleroderma of the head. J Neurol 1990;237:379-81.

6 Duyff RF, Vos J. A "scar" and epilepsy: coup de sabre. J Neurol Neurosurg Psychiatry 1998;65:568.

7 Dubeau F, Andermann F, Robitaille Y, et al. Morphea or focal scleroderma of the brain: intractable epilepsy and clinicopathologic correlation. Epilepsia 1988;29:712-13.

8 David J, Wilson J, Woo P. Scleroderma "en coup de sabre". Ann Rheum Dis 1991;50:260-2.

9 Liu P, Uziel Y, Chuang S, et al. Localized scleroderma: imaging features. Pediatr Radiol 1994;24:207-9

10 Menni S, Marzano AV, Passoni E. Neurologic abnormalities in two patients with facial hemiatrophy and sclerosis with morphea. Pediatr Dermatol 1997;4:113-16.

11 Kanzato N, Matsuzaki T, Komine Y, et al. Localized scleroderma associated with progressing ischemic stroke. J Neurol Sci 1999;163:86-9.

12 Terstegge K, Kunath B, Felber ST, et al. MR of brain involvement in progressive facial hemiatrophy (Romberg disease): reconsideration of a syndrome. Am J Neuroradiol 1994;15:145-50.

13 Yamanaka CT, Gibbs NF. Trauma-induced linear scleroderma. Cutis 1999;63:29-32.

14 Komocsi A, Tovari E, Kovacs J, et al. Physical injury as a provoking factor in three patients with scleroderma. Clin Exp Rheumatol 2000;18:622-4.

15 Pupillo G, Andermann F, Dubeau F. Linear scleroderma and intractable epilepsy: neuropathologic evidence for a chronic inflammatory process. Ann Neurol 1996;39:277-8

16 Hughes GR. Current understanding of systemic lupus erythematosus Inflammation 1984;8(suppl):S75-9.

17 Falanga V. Localized scleroderma. Med Clin North Am 1989;73:1 143-55.

18 Falanga V, Medsger TA, Reichlin M, et al. Linear scleroderma: clinical spectrum, prognosis and laboratory disorders. Ann Intern Med $1986 ; 104: 849-57$ 